\title{
Gegen Globalisierung und Demokratie. Die NPD als eine neue soziale Bewegung im europäischen Kontext?
}

\author{
Florian Hartleb
}

„Alles schmilzt unter der neuen Wüstensonne der Globalisierung in die politische Gestaltbarkeit(szumutung) zusammen. Alle gesellschaftlichen Akteure müssen darauf reagieren, darauf so oder so antworten; [...]." ${ }^{1}$ Ein nüchterner Parteienforscher kommt hingegen zu einem anderen Befund. Empirischen Untersuchungen zufolge wirkt die Globalisierung und sogar der supranationale Integrationsprozess auf das nationale Parteiensystem nur wenig. ${ }^{2}$ Allerdings finden in den EU-Mitgliedsländern euroskeptische und globalisierungskritische Positionen verstärkt Resonanz. ${ }^{3}$ Anton Pelinka kommt sogar zu folgendem Urteil: „Skepsis gegenüber der europäischen Integration bis hin zur prinzipiellen Ablehnung der Europäischen Union ist ein Phänomen, das die europäische Politik wesentlich beeinflusst. " 4

Die aktuelle NPD fungiert nach Ansicht des Bundesamtes für Verfassungsschutz als Gravitationsfeld im Rechtsextremismus ${ }^{5}$, womit ihr besondere Untersuchungsrelevanz gebührt. Mit ihrer Mischung aus vordergründig innovativem und reaktionärem Globalisierungsdiskurs erscheint sie als neue soziale Bewegung - ein Novum in der Geschichte des Rechtsextremismus in der Bundesrepublik Deutschland. Diese Behauptung mutet zunächst paradox an, gilt die NPD doch nach allgemeiner Auffassung als reaktionäre, ideologisch starre Kaderpartei. Um sie zu prüfen, sind zwei Fragen zu beantworten: Entspricht die NPD dem Typus einer neuen sozialen Bewegung? Wie antwortet die Partei auf die Globalisierung? Die Verknüpfung beider Fragen liegt in der Globalisierungsthematik. Der erste Fragekomplex stellt eher auf den Organisationsgrad, der zweite auf die Diskursebene ab, wobei es starke Interdependenzen gibt.

Die politikwissenschaftliche Rechtsextremismusforschung hat sich lange nicht mit den ideologischen Grundauffassungen hinter dem Vorhang der Globalisierungskritik beschäftigt. In erster Linie fragen die einschlägigen Publikationen danach, welche neue Mobilisierungsoder Wählerpotentiale sich durch die Globalisierung ergeben. Dietmar Loch und Wilhelm Heitmeyer zum Beispiel erkennen aufgrund der aktuellen sozialen Umbrüche Abwehrreak-

1 Ulrich Beck, Was ist Globalisierung?, Frankfurt am Main 2007, S. 13 f.

2 Vgl. Peter Mair, The Limited Impact of Europe on National Party Systems, in: WEP, 23. Jg. (2000), H. 4, S. 27 - 51. Siehe dazu auch Florian Grotz, Jenseits von Integrationsstudien und disziplinärer Neugründung: Ansätze zu einer europawissenschaftlich erweiterten Staats- und Regierungslehre, in: ZSE, 4. Jg. (2006), H. 3, S. 420 - 443, S. 436 f.

3 Mair selbst erkennt das nun und modifiziert damit seine Position. Vgl. Peter Mair, Political opposition and the European Union, in: Government and Opposition, 42. Jg. (2007), H. 1, S. 1 - 17. Vgl. auch André Krouwel / Koen Abts, Varieties of Euroscepticism and Populist Mobilization: Transforming Attitudes from Mild Euroscepticism to Harsh Eurocynicism, in: Acta Politica, 42. Jg. (2007), H. 2, S. $252-270$.

4 Anton Pelinka, Bestimmungsfaktoren des Euroskeptizismus. Zu den Ursachen der Krise der europäischen Integration, in: ders. / Fritz Plasser (Hrsg.), Europäisch Denken und Lehren. Festschrift für Heinrich Neisser, Innsbruck 2007, S. 233 - 247, S. 233.

5 Bundesamt für Verfassungsschutz (Hrsg.), Die „Nationaldemokratische Partei“ (NPD) als Gravitationsfeld im Rechtsextremismus, Köln 2006. 
tionen zugunsten rechtsautoritärer Vorstellungen. ${ }^{6}$ Hier sollen darüber hinausgehend auf der Akteursebene die Rechtsextremismus-, Globalisierungs- und Bewegungsforschung miteinander verknüpft werden. Der Bewegungsansatz erweitert die Perspektive hin zu einem kollektiven Handlungszusammenhang und berücksichtigt dynamische Entwicklungen. Die normative Extremismustheorie kann solche Antizipationseffekte nur bedingt leisten. Sie stützt sich als Bezugspunkt eher statisch, da staatszentriert auf den demokratischen Verfassungsstaat. ${ }^{7}$ Die Bewegungsforschung schließt vor allem an den Untersuchungsschwerpunkt der Ressourcenmobilisierung, der kollektiven Identität und der Deutungsmuster an. ${ }^{8}$ So betrachtet der folgende Beitrag die handlungszentrierten Intentionen des Akteurs „NPD“9 und die Suche nach Vernetzung. ${ }^{10}$ Die strategischen Neuorientierungen der Partei, die sich aus der ideologischen Verschiebung im deutschen Rechtsextremismus hin zum Antikapitalismus ergeben ${ }^{11}$, legen die Perspektive auf Globalisierungsprozesse und ihre Diskussion nahe, und zwar in ihrer wirtschaftlichen, politischen und kulturellen Dimension. ${ }^{12}$

\section{Die NPD - Gesellschaftliche Relevanz als neue soziale Bewegung?}

Die Diskussion, ob es eine neue soziale Bewegung „von rechts“ geben kann, ist keineswegs neu. Viele der Grundmuster, die Hans-Gerd Jaschke und Claus Leggewie bereits 1993 beziehungsweise 1994 entwickelt haben ${ }^{13}$, werden mit dem fraglichen Anwendungsfall der „neuen NPD“ wieder aktuell. 1994, als die neonazistischen Aktivitäten einen Höchststand erreichten, setzte eine rege Forschungsdiskussion darüber ein, die dann aber erstaunlich rasch verpuffte - auch weil die Aktivitäten zurückgingen. ${ }^{14}$ Nun bewegt sich der Stand der

6 Vgl. Dietmar Loch / Wilhelm Heitmeyer (Hrsg.), Schattenseiten der Globalisierung. Rechtsradikalismus, Rechtspopulismus und separatistischer Regionalismus in westlichen Demokratien, Frankfurt am Main 2001.

7 Vgl. Uwe Backes / Eckhard Jesse (Hrsg.), Vergleichende Extremismusforschung, Baden-Baden 2005.

8 Vgl. zu den Vorteilen des Bewegungsansatzes die Bemerkungen von Christoph Busch, Rechtsradikale Vernetzung im Internet, in: WeltTrends, 13. Jg. (2005), H. 48, S. 67 - 78, S. 67 f.

9 Vgl. dazu auch Fabian Virchow, Dimensionen der „Demonstrationspolitik“ der extremen Rechten in der Bundesrepublik Deutschland, in: Andreas Klärner / Michael Kohlstruck (Hrsg.), Moderner Rechtsextremismus in Deutschland, Hamburg 2006, S. 68 - 101, S. $69 \mathrm{ff}$.

10 Damit wird wissenschaftstheoretisch einem idiographischen, an einem Verstehen des Einzelfalls interessierten Vorgehen gefolgt. Idiographisch steht auf der Suche nach wissenschaftlichem Erkenntnisgewinn in einem (scheinbaren) Gegensatz zu einem nomothetischen Vorgehen. Vgl. Detlef Jahn, Einführung in die vergleichende Politikwissenschaft, Wiesbaden 2006, S. 163.

$11 \mathrm{Vgl}$. Bernd Sommer, Anti-capitalism in the name of ethno-nationalism: ideological shifts on the German extreme right, in: Patterns of Prejudice, 42. Jg. (2008), H. 3, S. $305-316$.

12 Vgl. auch die Studie des namhaften Extremismusforschers Cas Mudde, Populist Radical Right Parties in Europe, Cambridge 2007, S. 185 - 197. Zudem Claus Leggewie, Rechts gegen Globalisierung, in: IP, 58. Jg. (2003), H. 4, S. $33-40$.

13 Vgl. Hans-Gerd Jaschke, Rechtsradikalismus als soziale Bewegung. Was heißt das?, in: Vorgänge, 32. Jg. (1993), H. 122, S. 105 - 116; Claus Leggewie, Rechtsextremismus - eine neue soziale Bewegung?, in: Wolfgang Kowalsky / Wolfgang Schroeder (Hrsg.), Rechtsextremismus. Einführung und Forschungsbilanz, Opladen 1994, S. 325 - 338.

14 Vgl. zur Kritik Thomas Ohlemacher, Schmerzhafte Episoden: Wider die Rede von einer rechten Bewegung im wiedervereinigten Deutschland, in: Forschungsjournal Neue Soziale Bewegungen, 7. Jg. (1994), H. 4, S. $16-25$. 
neonazistischen Kameradschaften im „neuen Umfeld“ der NPD wieder auf dem Niveau von Mitte der neunziger Jahre. ${ }^{15}$ Lässt sich mit Blick auf diese quantitative Parallele von der NPD als neue soziale Bewegung sprechen? Manche Autoren schließen dies für die Organisation des Rechtsextremismus kategorisch aus, da die aufgegriffenen Themen nicht den bewegungstypischen entsprechen würden. ${ }^{16}$ Mit dem Globalisierungsthema ist dieser Einwand jedoch wenig plausibel. Noch weiter geht die Ansicht, allen neuen sozialen Bewegungen seien postmaterialistische Wertevorstellungen gemeinsam. Dann könnte der Rechtsextremismus per definitionem keine neue soziale Bewegung sein. ${ }^{17}$

\subsection{Zum Begriff der neuen sozialen Bewegung}

Die Bezeichnung der neuen sozialen Bewegung entstand bei der sozialwissenschaftlichen Analyse von Protestbewegungen in den westlichen Demokratien, in der Bundesrepublik im Zuge der Friedens- und Umweltbewegung der siebziger und achtziger Jahre. Immer noch prägend für die Diskussion ist die Definition von Joachim Raschke aus dem Jahr 1985. Er sieht die neue soziale Bewegung als mobilisierenden kollektiven Akteur, der mit hoch symbolischer Aktion grundlegenden sozialen Wandel herbeiführen, verhindern oder rückgängig machen will. ${ }^{18}$ Darunter lassen sich auch expressis verbis rückwärtsgewandte wie antidemokratisch ausgerichtete Positionen subsumieren. Demnach könnte der Begriff auf ein politisches und soziales Phänomen wie den Rechtsextremismus angewandt werden.

Neue soziale Bewegungen entstehen in Zeiten gesellschaftlicher Umbrüche oder Krisen. Sie haben Netzwerkcharakter und grenzen sich von strukturierten Organisationen ab. Festgefügte Organisationen können allerdings Teilakteure in solchen sozialen Bewegungen sein. Im Unterschied zu spontanen Massenkundgebungen hat das In-Bewegung-Bleiben einen dauerhaften Charakter. ${ }^{19}$ Kollektive Identitäten gelten beim Bewegungsansatz als Kitt für gemeinsames oppositionelles Handeln und sind von gewisser Stabilität. Es entwickelt sich durch den Appell an Emotionen ein starkes Wir-Gefühl. Habitus, Riten, Kleidung und emotionale Bindungen gelten als charakteristisch. ${ }^{20}$ Symbolik ist von großer Bedeutung.

15 Vgl. zur Reorganisation Martin Thein, Der organisatorische Erneuerungsprozess als Bedingung für den Aufschwung des Neonazismus - Eine vergleichende Betrachtung traditioneller Organisationsstrukturen und der „freien Kameradschaften“, in: Uwe Backes / Henrik Steglich (Hrsg.), Die NPD - Erfolgsbedingungen einer rechtsextremistischen Partei, Baden-Baden 2007, S. 211 -231 .

16 Vgl. Roland Roth, ,Patch-work'. Kollektive Identitäten neuer sozialer Bewegungen, in: Kai-Uwe Hellmann / Ruud Koopmans (Hrsg.), Paradigmen der Bewegungsforschung, Opladen / Wiesbaden 1998, S. $51-69$.

17 Vgl. zusammenfassend Hermann Adam, Bausteine der Politik. Eine Einführung, Wiesbaden 2007, S. 146 f.

18 Vgl. Joachim Raschke, Soziale Bewegungen. Ein historisch-systematischer Grundriss, Frankfurt am Main 1985, S. 77.

19 Vgl. wegweisend Armin Pfahl-Traughber, Droht die Herausbildung einer Antiglobalisierung von rechtsextremistischer Seite? Globalisierung als Agitationsthema des organisierten Rechtsextremismus, in: Bundesministerium des Innern (Hrsg.), Extremismus in Deutschland. Erscheinungsformen und aktuelle Bestandsaufnahme, Berlin 2004, S. 98 - 135, S. 124.

20 Vgl. Roland Roth, a.a.O. (Fn. 16), S. $51-69$. 


\subsection{Zum Erscheinungsbild der NPD in den neuen Bundesländern}

Die NPD hat ihr Erscheinungsbild unter dem Parteivorsitzenden Udo Voigt (seit 1996) stark verändert. Der ehemalige Bundeswehrhauptmann und studierte Politologe zog die Konsequenz aus der Bedeutungslosigkeit der Partei. Langsam und schrittweise öffnete er sie für die neonationalsozialistische Szene, mit dem Ziel, ein „kollektives Ganzes“ zu verkörpern. Bedeutsam wurde nun auch die Nachwuchsrekrutierung ebenso wie die Konzentration auf regionale Schwerpunkte in Ostdeutschland, zunächst Sachsen und mit dem Umweg der Kameradschaftsszene auch das eher ländlich strukturierte Mecklenburg-Vorpommern. Modernisierungskrisen durch Perspektivlosigkeit, hohe Arbeitslosigkeit, damit einhergehende Demokratieunzufriedenheit und Sehnsucht nach kollektiver Identität bieten hier konkrete Anknüpfungspunkte. ${ }^{21}$

Der sächsische Wahlerfolg von 2004, der den Einzug in den Landtag bedeutete, wurde schon 1999 durch Umzüge von Personen wie auch der Parteizeitung von West nach Ost vorbereitet. Zahlreiche Kader wie Holger Apfel, inzwischen NPD-Fraktionsvorsitzender im Sächsischen Landtag, konnten gewonnen werden. In Mecklenburg-Vorpommern, wo der NPD 2006 der zweite Landtagseinzug glückte, lassen sich ebenfalls diese frühzeitigen „Importe“ nachweisen.

Mittlerweile nutzt die NPD auch die für soziale Bewegungen typischen Aktionsformen wie Demonstrationen. Sie setzt sich einen symbolischen Rahmen, stilisiert sich zum Retter der Not und benennt Schuldige. ${ }^{22}$ Die Partei agiert dabei gegen das System. Unverblümt schreibt sie: „Eine neue soziale Bewegung muss die bestehenden Verhältnisse überwinden. "23 Damit gelingt ihr vor allem in Mecklenburg-Vorpommern der Brückenschlag zu den Kameradschaften und zur militanten Szene. Die Partei hat dort nur wenige Mitglieder ${ }^{24}$, kann aber auf rechtsextremistische Strukturen aufbauen. Auf der NPD-Landtagsliste 2006 stand mit Tino Müller ein Kandidat auf Platz 2, der vorher eine Bürgerinitiative gegen Asylbewerberwohnheime ins Leben gerufen hatte („Schöner und sicherer wohnen“). Müller fungiert als wichtiger Verbindungsmann zwischen Partei und Kameradschaftsszene. Das gilt auch für andere Landesverbände wie in Berlin: „Es besteht für die NPD die erhebliche Gefahr, dass die Neonazis der freien Kameradschaftsszene angesichts der Schwäche der ursprünglichen Parteiorganisation die Partei selbst übernehmen. "25 Teilweise kam dieser Schritt von der bewegungsförmigen Szene hin zur Aktivität unter dem Deckmantel der Partei als Spätfolge zahlreicher Vereinsverbote zwischen 1992 und 1995 zustande. Zwei Protagonisten stehen exemplarisch dafür: Thorsten Heise, seit 2004 bei der NPD, war früher Mitglied der

21 Vgl. Rainer Fromm, Schwarze Geister, Neue Nazis. Jugendliche im Visier totalitärer Bewegungen, München 2008, S. 225 - 299.

22 Vgl. Lazaros Miliopoulos, Strategische Ansätze, Potentiale und Perspektiven der NPD, in: Uwe Backes / Henrik Steglich (Hrsg.), a.a.O. (Fn. 15), S. 121 - 141, S. 124.

23 Vgl. Arbeitskreis Wirtschaftspolitik beim NPD-Parteivorstand, Grundlagen einer raumorientierten Volkswirtschaftslehre, Profil-Broschüre Nr. 13, Berlin 2006, S. 78.

24 Lediglich um die 200 Mitglieder hat die NPD in dem Flächenstaat.

25 Christian Demuth / Sarah Ganter, Die rechtsradikalen und rechtsextremistischen Parteien in Berlin, in: Christian Junge / Jakob Lempp (Hrsg.), Parteien in Berlin, Berlin 2007, S. 155 - 175, S. 171. Das gilt auch für Sachsen. Vgl. dazu Marc Brandstetter, Die NPD in Sachsen: Politische Struktur und gesellschaftliche Verwurzelung, in: ZParl, 38. Jg. (2007), H. 2, S. 349 - 367. 
1995 verbotenen Freiheitlichen Arbeiterpartei (FAP). Für Thomas Wulff, einst unter anderem bei der ebenfalls 1995 verbotenen Nationalen Liste (NL) aktiv, gilt dasselbe. ${ }^{26}$ Die meisten Forscher konnten sich noch Anfang und Mitte der neunziger Jahre diese grundsätzliche Wandlung im Rechtsextremismus nicht vorstellen. ${ }^{27}$

Bedeutsam für die Einordnung als neue soziale Bewegung ist neben der eigenen quantitativen Stärke die Verankerung in der Bevölkerung. Dafür ist ein Eindringen in gesellschaftliche Scharnierorganisationen, die so genannten sozialen Relais notwendig. So schaffte es die Friedensbewegung, Ende der siebziger und Anfang der achtziger Jahre Akzeptanz an Universitäten zu finden und gesellschaftlichen Einfluss auszuüben. Das gelang mit straffer Organisation, Medienwirksamkeit und einem festen Demonstrationszyklus. ${ }^{28}$ Scharnierorganisationen wie Gewerkschaften, Kirchen oder Universitäten fehlen der NPD. Ein gewisses soziales Einfallstor findet sich gleichwohl bei einem Segment an jungen Männern mit formal geringer Bildung und einem niedrigen Sozialstatus. Das zeigte sich bei der Landtagswahl 2006 in Mecklenburg-Vorpommern: Wie in Sachsen erzielte die NPD bei den 18- bis 24-Jährigen ihr bestes Ergebnis. ${ }^{29}$

Der Journalist Toralf Staud spricht von der „Faschisierung der ostdeutschen Provinz“30. Selbst dort ist sie aber entgegen medialer Effekthascherei nicht in der Mitte der Gesellschaft angekommen. Auf kommunaler Ebene lastet, wie in Mecklenburg-Vorpommern, die tagtägliche Arbeit auf wenigen Einzelpersonen. ${ }^{31}$ Die militante Straßenmobilisierung der NPD zeugt nicht von breiter gesellschaftlicher Verankerung, sondern führt fast stets zu massiven Gegenveranstaltungen und Protesten oder stößt zum Teil auf rechtliche Schranken. Generell mangelt es in der Bundesrepublik nicht an Mitteln der Repression. Es existiert eine Vielzahl an gesetzlichen Bestimmungen, beispielsweise gegen das Tragen nationalsozialistischer Abzeichen, den Hitlergruß oder die Verbreitung von NS-Schriftgut. ${ }^{32}$ Daneben gibt es als schärfstes Schwert der streitbaren Demokratie die Möglichkeit des Parteiverbots: Es wurde gegen die NPD vor ihrer neuen Bedeutung Ende Januar 2001 zwar gezückt, wurde aber durch das formell bedingte Scheitern im März 2003 zu einer stumpfen

26 Vgl. genauer Julia Gerlach, Auswirkungen der Verbote rechtsextremistischer Vereine auf die NPD, in: Uwe Backes / Henrik Steglich (Hrsg.), a.a.O. (Fn. 15), S. $233-260$.

27 Vgl. nur Ruud Koopmans, Soziale Bewegung von rechts? Zur Bewegungsförmigkeit rechtsradikaler und ausländerfeindlicher Mobilisierung in Deutschland, in: Jens Mecklenburg (Hrsg.), Handbuch deutscher Rechtsextremismus, Berlin 1996, S. 767 - 781, S. 779.

28 Vgl. Florian Hartleb, Die westdeutsche Friedensbewegung. Entstehung, Entwicklung und Unterwanderungsversuche, in: Eckhard Jesse / Günther Heydemann (Hrsg.), 15 Jahre deutsche Einheit. Deutsch-deutsche Begegnungen, deutsch-deutsche Beziehungen, Berlin 2006, S. 159 - 175.

29 Vgl. Sandra Pingel-Schliemann / Karl-Georg Obse, Der Wahlerfolg der NPD in MecklenburgVorpommern, in: Deutschland Archiv, 39. Jg. (2006), H. 6, S. 968 - 972, S. 971. Vgl. zu Fakten im NPD-Wahlkampf, Innenministerium Mecklenburg-Vorpommern (Hrsg.), Verfassungsschutzbericht 2006, Schwerin 2007, S. $71-77$.

30 Vgl. Toralf Staud, Moderne Nazis. Die neuen Rechten und der Aufstieg der NPD, Köln 2005.

31 Vgl. Hubertus Buchstein / Benjamin Fischer, Die NPD in den kommunalen Parlamenten Mecklenburg-Vorpommerns, in: Uwe Backes / Henrik Steglich (Hrsg.), a.a.O. (Fn. 15), S. 143 - 165, S. 154.

32 Diesen Punkt gibt Frank Decker in einer Analyse der Bekämpfungsstrategien im Umgang mit der NPD zu bedenken. Vgl. ders., Verbieten und vergessen? Politische Perspektiven im Kampf gegen Rechts, in: Mut, 43. Jg. (2008), H. 485, S. 28 - 38, S. 34. 
Waffe, welche der streitbaren Demokratie selbst Schaden zufügte. ${ }^{33}$ Insgesamt sorgen aber die „Schatten der Vergangenheit“ dafür, dass jegliches Aufkommen rechtsextremistischer Tendenzen von der Öffentlichkeit kritisch geahndet wird. ${ }^{34}$

Die Beurteilung der NPD mit Blick auf den Bewegungscharakter kommt damit zu folgendem Ergebnis: (1) Es ist eine Infiltration nach dem für soziale Bewegungen typischen Kosten-Nutzen-Kalkül festzustellen; keine breite, sondern gezielt-begrenzte gesellschaftliche Verankerung wird angestrebt („Faschisierung der „ostdeutschen Provinz“, vor allem im jungen Alterssegment); (2) Solidarisierung findet im rechtsextremistischen Lager in Form des Brückenschlags der „neuen“ NPD zu den Kameradschaften und freien Kräften statt (mittelbare Folge zahlreicher Vereinsverbote in den 1990er Jahren); (3) nach außen präsentiert sich die Partei punktuell, verleiht ihrer kollektiven Identität Ausdruck und agiert betont symbolisch (Demonstrationen, die wiederum massive Gegendemonstrationen und damit Öffentlichkeit auf sich ziehen).

\section{Die NPD und die Globalisierung}

Der Globalisierungsdiskurs ist der Ideologieverstärker der NPD. Seit 2004 ist sie nach ihrem Einzug in zwei Landtage und dem offenen Schulterschluss mit den Kameradschaften dominant im rechtsextremistischen Lager geworden. ${ }^{35}$ Die Verfassungsschutzbehörden sprechen neuerdings von der NPD als "Gravitationsfeld im Rechtsextremismus“36. Der Themenblock „Wirtschaft und Soziales“ im internationalen Kontext hat für die NPD von heute große Bedeutung. Schlüsselfigur für die NPD ist der sächsische Landtagsabgeordnete und Historiker Jürgen W. Gansel. ${ }^{37}$ Er forciert die inhaltliche Akzentverschiebung hin zur Sozialpolitik und zum aktiven Aufgreifen des Globalisierungsdiskurses.

Dies lässt sich an drei Beispielen zeigen: Erstens entfachte die Parteizeitung „Deutsche Stimme“ im April 2003 eine „Querfront-Debatte“ um die Frage „Sind Rechte und Linke zu einer Allianz fähig? Mit einer sozialen, ökologischen und völkischen Bewegung das System der Globalisierer bekämpfen“. Vor allem der gemeinsame Gegner lege eine Zusam-

33 Vgl. dazu mit einem signifikanten Titel Lars Flemming, Das NPD-Verbotsverfahren. Vom „Aufstand der Anständigen“ zum „Aufstand der Unfähigen“, Baden-Baden 2005. Im europäischen Vergleich ist diese Praxis des Parteiverbots keineswegs Usus. Vgl. Katerina Kontopodi, Die Rechtssprechung des Europäischen Gerichtshofs für Menschenrechte zum Verbot politischer Parteien, Baden-Baden 2007.

34 Vgl. unter anderem Frank Decker / Florian Hartleb, Populism on Difficult Terrain: The Rightand Left-Wing Challenger Parties in the Federal Republic of Germany, in: German Politics, 16. Jg. (2007), H. 4, S. $434-454$, S. 442 ff.

35 So auch der Titel einer Studie zur NPD von heute. Vgl. Harald Bergsdorf, Die neue NPD. Antidemokraten im Aufwind, München 2007. Etwas effekthascherisch Andrea Röpke / Andreas Speit (Hrsg.), Neonazis in Nadelstreifen. Die NPD auf dem Weg in die Mitte der Gesellschaft, Berlin 2008 .

36 Vgl. Bundesamt für Verfassungsschutz (Hrsg.), a.a.O. (Fn. 5).

37 Vgl. nur seine Artikel in der Parteizeitung „Deutsche Stimme“. Zur zentralen Bedeutung des Organs für Entwicklung, Ideologie und Strategie der NPD vgl. Florian Hartleb, Die „Deutsche Stimme“ - Das intellektuelle Sprachrohr der NPD?, in: Uwe Backes / Henrik Steglich (Hrsg.), a.a.O. (Fn. 15), S. 355 - 382. 
menarbeit nahe. ${ }^{38}$ Zweitens bemühte Gansel im Oktober 2007 das Bild des Hufeisenmodells, um die Nähe zwischen „rechts“ und „links“ an den Polen aufzuzeigen: „Übergreifende Einstellungen in Folge des Globalisierungsangriffs auf National- und Sozialstaat machen politische Richtungsbegriffe obsolet." ${ }^{39}$ Drittens appellierte er an das Kollektiv der Globalisierungsgegner: „Reißen wir den Globalisten und Multikulturalisten ihre pseudohumanitäre Masken vom Gesicht. Entlarven wir sie als Wegbereiter einer Einzeitgesellschaft, die den Menschen das verweigert, wonach sie hungern: soziale Teilhabe, Gerechtigkeit und Sicherheit einerseits, nationale Solidarität, Identität und Gemeinschaft andererseits. " ${ }^{40}$ Die Partei sucht den Schulterschluss mit linken Globalisierungsgegnern, kopiert die Argumentation und verbrämt sie mit der eigenen Ideologie. Damit steht sie im europäischen Kontext nicht allein. So schließen sich beispielsweise flämische Nationalisten immer wieder globalisierungskritischen Demonstrationszügen an.

Das grenzüberschreitende Andocken der NPD erreichte beim G8-Gipfel in Heiligendamm 2007 einen neuen Höhepunkt. Die Partei wollte den öffentlichen Blick auf die Globalisierungsproteste für sich nutzen - mit eingängigen Parolen wie „Zukunft statt Globalisierung - Arbeit für Millionen statt Profit für Millionäre“ ${ }^{41}$. Die linken Globalisierungsgegner zeigten die kalte Schulter. Eine Gruppierung wie Attac mit Schwerpunkten in Frankreich und Deutschland zog schon seit ihrer Entstehung als globalisierungskritisches Netzwerk einen Trennungsstrich: „Ausgeschlossen sind Neofaschisten, die immer mal wieder versuchen, mit nationalistisch, gar antisemitisch motivierter Kapitalismuskritik bei Attac unterzuschlüpfen. Für Rassismus, Antisemitismus, Fremdenfeindlichkeit, Chauvinismus und verwandte Ideologien gibt es keinen Platz. " 42

Aber nicht nur wegen der Distanz der linken Globalisierungsgegner war das Unterfangen weitgehend erfolglos. Innere Widersprüche blieben nicht aus. Udo Pastörs schlug als Fraktionsvorsitzender frühzeitig vor, der G8-Gipfel solle am besten in einer Raumstation stattfinden. Mecklenburg-Vorpommern brauche keinen „Globalisierungs-Gleichschaltungs-

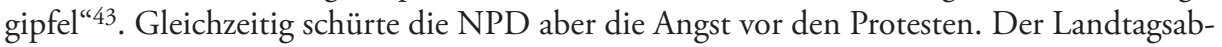
geordnete Michael Andrejeweski appellierte frühzeitig an den Rechtsstaat und die Landesregierung zum Schutz vor „ausländischen Gewalttouristen“44. Nach den Ausschreitungen am Rande der Demonstrationen am 2. Juni in Rostock stellte der Landesvorstand der NPD

38 Vgl. den Beitrag mit dem oben genannten Titel von Roland Wuttke, in: Deutsche Stimme, 28. Jg. (2004), H. 4, S. 21.

39 Jürgen W. Gansel, National versus international. In der Globalisierungsära verlieren die Begriffe „links" und „rechts“ ihre Aussagekraft, in: Deutsche Stimme, 31. Jg. (2007), H. 10, S. 6.

40 Jürgen W. Gansel, Die Stunde einer nationalen Solidar- und Gerechtigkeitsbewegung ist da. Gedanken zu einem zeitlosen Identifikationsangebot für die Globalisierungsopfer, in: Deutsche Stimme, 32. Jg. (2008), H. 1, S. 22.

41 Vgl. Lisa Erdmann, G-8-Gipfel. Wie Rechtsradikale in Heiligendamm punkten wollen, in: Spiegel online vom 23. April 2007, www.spiegel.de/deutschland/0,1518,478500,00.html.

42 Christian Grefe / Mathias Greffrath / Harald Schumann, Attac. Was wollen die Globalisierungskritiker?, Berlin 2002, S. 137.

43 Vgl. Udo Pastörs, G8-Gipfel am besten in Raumstation. Redemanuskript aus der Debatte zum Nachtragshaushalt für den G8-Gipfel. Rede gehalten am 15. November 2006 in der 6. Sitzung des Landtages von Mecklenburg-Vorpommern.

44 Vgl. Michael Andrejeweski, Den Rechtsstaat durchsetzen. Redemanuskript zum Thema: Sicherheitsfragen im Zusammenhang mit dem G8-Gipfel. Rede gehalten am 15. November 2006 in der 6. Sitzung des Landtages von Mecklenburg-Vorpommern. 
eine umfassende Anfrage an die Landesregierung: Gesetze sollen es möglich machen, „bei zukünftigen gewalttätigen Ausschreitungen Gummigeschosse einzusetzen “45. Die antidemokratische NPD sah sich aus taktischen Motiven nun als Schützer des Rechtsstaats.

Der Globalisierungsdiskurs der NPD ist nicht neu im Sinne einer Loslösung von der nationalsozialistischen oder faschistischen Ideologie. Die als Alternative zur Globalisierung propagierte Volksgemeinschaft wurde von der NSDAP zwar nicht erfunden, aber doch entscheidend geprägt. Der Nationalsozialismus nahm für sich in Anspruch, eine Blut-, Rassenund Schicksalsgemeinschaft zu sein und damit den Einzelnen „total“ zu erfassen. Udo Voigt kokettiert mit Adolf Hitlers Gedankengängen aus „Mein Kampf“. So forderte er schon 1998 die „Überwindung der kapitalistischen Zinswirtschaft “46, eine Wortwahl Hitlers ${ }^{47}$, der mit einem völkischen Erklärungsmodell als Antwort auf die Globalisierung argumentierte. Auch die NPD sieht alle drei Dimensionen der Globalisierung aus der Perspektive der Volksgemeinschaft.

\subsection{Wirtschaftliche Dimension}

Die wirtschaftliche Globalisierung führt zu einem erheblichen Standortwettbewerb und starker Konkurrenz. Nationalstaaten verlieren traditionelle Regelungskompetenzen, transnational operierende Großkonzerne können, so das negative Szenario, verschiedene Standorte gegeneinander ausspielen, zu Lohndumping greifen. ${ }^{48}$ Rechtsextreme Akteure antworten darauf heute mit einer scharfen Kapitalismuskritik. In den achtziger und frühen neunziger Jahren vertraten viele erfolgreiche europäische Rechtsaußenparteien noch neoliberale, hingegen kaum kapitalismuskritische Positionen. Beispiele sind die FPÖ und der Front National. Der Wettbewerbsgedanke dominierte. Heute buhlen solche rechten Formationen um Globalisierungsverlierer. Elektoral ist das veränderte Angebot durch den größeren Anteil an gering qualifizierten Arbeitnehmern und Arbeitslosen nur konsequent.

Neben der Globalisierungsverliererthese gibt es als griffige Erklärungsformel den Wohl-

45 Vgl. Drs. 5 vom 27. Juni 2007, Große Anfrage der NPD-Fraktion: G8-Gipfel in Heiligendamm 2007, S. 8.

46 Laut einer Sonderausgabe der Deutschen Stimme, 22. Jg. (1998), H. 5. Vgl. auch einen anderen Artikel zur Arbeitslosigkeit, Lothar Ehrlichmann, Zinsgeldsystem als Motor der Arbeitsplatzvernichtung, in: Deutsche Stimme, 28. Jg. (2004), H. 7, S. 9.

47 „Als ich den ersten Vortrag Gottfried Feders über die ,Brechung der Zinsknechtschaft' anhörte, wusste ich sofort, dass es sich um eine theoretische Wahrheit handelte, die von immenser Bedeutung für die Zukunft des deutschen Volkes werden müsste. Die scharfe Scheidung des Börsenkapitals von der nationalen Wirtschaft bot die Möglichkeit, der Verinternationalisierung der deutschen Wirtschaft entgegenzutreten, ohne zugleich mit dem Kampf gegen das Kapital überhaupt die Grundlage einer unabhängigen völkischen Selbsterhaltung zu bedrohen. Mir stand die Entwicklung Deutschlands schon viel zu klar vor Augen, als dass ich nicht gewusst hätte, dass der schwerste Kampf nicht mehr gegen die feindlichen Völker, sondern gegen das internationale Kapital ausgefochten werden muss." Adolf Hitler, Mein Kampf, München 1941, S. 232 f. Wie Barbara Zehnpfennig in einer überzeugenden Analyse nachweist, ist das Werk in toto entgegen der verbreiteten Meinung kein geistiges Wirrwarr, sondern ein in sich logisches Gedankengebäude, das Hitlers innenpolitischem Fahrplan wie seinem außenpolitischen Programm Grund und Ziel gab. Vgl. Barbara Zehnpfennig, Hitlers Mein Kampf. Eine Interpretation, München 2000.

48 Vgl. Klaus Dörre, Globalisierung - Ende des rheinischen Kapitalismus, in: Dietmar Loch / Wilhelm Heitmeyer (Hrsg.), a.a.O. (Fn. 6), S. 63 - 90. 
fahrtschauvinismus, der die Leistungen des Wohlfahrtsstaats der einheimischen Bevölkerung vorbehalten will. Wohlfahrt bezieht sich dabei auf die vom Staat erbrachten Sozialleistungen für den Einzelnen, nicht auf den gesellschaftlichen Wohlstand. ${ }^{49}$ Es wird mit der Fiktion argumentiert, Verteilungskonflikte drehten sich ausschließlich um den eigenen Nationalstaat. Rechtsextremistische Formationen bedienen mit Abschottungsparolen Schutzbedürfnisse der Bevölkerung. Sie schüren die Angst vor ökonomischer Deklassierung und sozialem Abstieg.

Schon 1997 stellte die NPD in ihrem Parteiprogramm ausführlich klar: Die Globalisierung der Wirtschaft führt zur Ausbeutung der Erde und Massenerwerbslosigkeit. ${ }^{50}$ Für Mecklenburg-Vorpommern baute die Partei eine fundamentale Gegenposition zur wirtschaftlichen Globalisierung im Ostseeraum auf und reagierte im Wahlprogramm von 2006 auf den jüngsten regionalen Globalisierungsschub. Durch die Osterweiterung liegt das Land nicht mehr am Rand, sondern im Zentrum der Europäischen Union. Die Ostsee ist handelsstrategisch das verbindende Element, nicht mehr nur zu den skandinavischen EUMitgliedern, sondern auch zu Polen und den baltischen Staaten. Die NPD, sonst stolz auf die deutsche Geschichte, ignoriert die Tradition der Hanse. Vielmehr schrieben die Rechtsextremisten: „Deutschland muss wieder deutsch sein. Die NPD ist die einzige konsequente Kraft gegen Globalismus und für eine volks- und raumnahe Wirtschaft! Sie ist die Partei, die sich bewusst für das Existenzrecht der Völker einsetzt und gegen die Zerstörung der Volksidentitäten kämpft, die sowohl Ab- wie auch Zuwanderung großer Teile der Arbeitnehmerschaft automatisch mit sich bringen. "51

Die Partei bedient also gerade nicht die neoliberale Agenda und widerlegt damit die Befunde der vergleichenden Parteienforschung. Herbert Kitschelt machte 1995 in seiner renommierten komparatistischen Studie die neoliberale Konzeption als Erfolgsbedingung rechter Flügelparteien in Westeuropa aus. ${ }^{52}$ Erst kürzlich verteidigte er seine Position. ${ }^{53}$ Die NPD greift hingegen zu einem scharfen Antikapitalismus, propagiert wirtschaftliche Abschottung, Protektionismus und Autarkie. Sie erläutert das vage Konzept der raumorientierten Volkswirtschaft neuerdings mit breiten ideengeschichtlichen Anleihen, beispielsweise auf den Merkantilismus, Johann Gottlieb Fichte oder Georg Wilhelm Friedrich Hegel, selbst auf Karl Marx. Dieser hätte eine bis heute zutreffende Beschreibung des Globalisierungsprozesses geliefert. ${ }^{54}$ Die raumorientierte Volkswirtschaft beruht auf dem Prinzip der Volksgemeinschaft. Das „Wie“ ihrer Umsetzung bleibt trotz des neuartigen Rückgriffs auf die

49 Vgl. Frank Decker, Der neue Rechtspopulismus, Opladen 2004, S. 198 - 206.

50 Vgl. Nationaldemokratische Partei Deutschlands (Hrsg.), Parteiprogramm, Stuttgart 1997.

51 Vgl. Aktionsprogramm der NPD zur Landtagswahl 2006.

52 Vgl. Herbert Kitschelt / Anthony J. McGann, The Radical Right in Western Europe. A Comparative Analysis, Ann Arbor 1995. Seither finden eingehende Diskussionen über das Buch statt, welches 1996 den bekannten Woodrow Wilson Foundation Award der American Political Science Association erhielt. Vgl. nur Steffen Kailitz, Das ideologische Profil rechter (und linker) Flügelparteien in den westeuropäischen Demokratien - Eine Auseinandersetzung mit den Thesen Herbert Kitschelts, in: Uwe Backes / Eckhard Jesse (Hrsg.), Gefährdungen der Freiheit. Extremistische Ideologien im Vergleich, Göttingen 2006, S. 283 - 320.

53 Vgl. Herbert Kitschelt, Review Article. Growth and Persistence of the Radical Right in Postindustrial Democracies: Advances and Challenges in Comparative Research, in: WEP, 30. Jg. (2007), H. 5, S. $1176-1206$.

54 Vgl. Arbeitskreis Wirtschaftspolitik beim NPD-Parteivorstand, a.a.O. (Fn. 23). 
Geschichte offen. Als unzutreffend erweist sich daher an diesem Beispiel die These, der europäische Faschismus habe sich durch eine neuartige neoliberale Ausrichtung erfolgreich modernisiert. ${ }^{55}$

Flankiert wird der ökonomische Antiglobalisierungsdiskurs der NPD von alten antisemitischen Stereotypen, die eine globale jüdische Herrschaft des Finanzkapitals behaupten ${ }^{56}$, die die Volksgemeinschaft bedrohe.

\subsection{Politische Dimension}

Im Kontext des europäischen Rechtsextremismus spielt die europäische Integration als Form von Global Governance eine besondere Rolle. Die Institutionen der EU sind, so lautet der Vorwurf, nicht nur wenig transparent, sondern leiden oftmals an einem Legitimitätsproblem in Gestalt eines Demokratiedefizits. Der EU fehlen ein europäischer demos und eine europäische Öffentlichkeit. Daraus ergeben sich strukturelle Hindernisse für eine volle Parlamentarisierung. Gerade das Europäische Parlament (EP) ist keine Vertretung eines europäischen Volkes: Nationale Parteien konkurrieren in nationalen Wahlkämpfen um nationale Kontingente. ${ }^{57}$ Weil die nationale Opposition im Europäischen Rat und Ministerrat über keinerlei Einfluss verfügt, ist sie leicht versucht, gegen die „Eurokraten“ zu wettern. An diesem wunden Punkt der Legitimitätsfrage finden auch (fundamental-)oppositionelle rechtsextremistische Parteien Anknüpfungspunkte und Mobilisierungsmöglichkeiten. ${ }^{58}$ Mit Blick auf ihren Euroskeptizismus gilt es, zwischen einer „weichen“ und einer „harten“ Variante zu unterscheiden. Ersterer, der keineswegs extremistisch sein muss, beinhaltet die qualifizierte Ablehnung bestimmter Aspekte des Integrationsprojektes oder der EU in ihrer gegenwärtigen institutionellen Form. „Harter“ Euroskeptizismus lehnt die „Idee Europa“ aus prinzipiellen Gründen ab. ${ }^{59}$ Rechtsextremisten sind meist „harte“ Euro-

55 Vgl. Christina Kaindl, Völkischer Antikapitalismus: Globalisierungskritik von rechts?, in: Richard Gebhardt (Hrsg.), Rosen auf den Weg gestreut. Deutschland und seine Neonazis, Köln 2007, S. 139 - 150, S. $148 \mathrm{f}$.

56 Ein Aufsatz in der Parteizeitung beschäftigt sich mit dem „Globalkapitalismus“: „Für Philosemiten und einschlägige Interessenvertreter ist es eine unausrottbare Verschwörungstheorie, für andere aber eine schlichte Wahrheit [...], die durch das reale Weltgeschehen immer wieder Bestätigung findet: die Schlüsselstellung von Juden in den privaten und staatlichen Machtzentren des Weltkapitalismus. Neben der US-Notenbank und der Weltbank wird demnächst auch das EUHandelskommissariat in auserwählter Hand sein. Alles ein Zufall?" Thoralf Trenkmann, Globalkapitalismus. Jüdisches Finanztrio bald komplett, in: Deutsche Stimme, 28. Jg. (2004), H. 10, S. 18. Vgl. dazu auch Fabian Virchow, Die extreme Rechte als globalisierungskritische Bewegung?, in: Ivonne Bemerburg / Arne Niederbacher (Hrsg.), Die Globalisierung und ihre Kritik(er). Zum Stand der aktuellen Globalisierungsdebatte, Wiesbaden 2007, S. 215 - 232.

57 Vgl. unter anderem Gerd Strohmeier, Die EU zwischen Legitimität und Effektivität, in: APuZ, B 10 (2007), S. $24-30$, S. 29 f.

58 Vgl. Thomas Greven, Rechtsextreme Globalisierungskritik, in: ders. / Thomas Grumke (Hrsg.), Globalisierter Rechtsextremismus? Die extremistische Ära in der Ära der Globalisierung, Wiesbaden 2006, S. $15-29$, S. $19-21$.

59 Vgl. Paul Taggart / Aleks Szczerbiak, Contemporary Euroscepticism in the party systems of the European Union candidate states of Central and Eastern Europe, in: European Journal of Political Research, 43. Jg. (2004), H. 1, S. 1 - 27, S. 3 f. Zu weiteren Details, mit Bezug auf Taggart und Szczerbiak vgl. Guido Tiemann, Euroskeptische Parteien zwischen Strategie und Ideologie, in: 
skeptiker: Sie wollen ein Europa der ethnisch homogenen Nationalstaaten und sind gegen das EU-Europa. Ethnische und politische Einheit sollen zwei Seiten derselben Medaille sein.

Entsprechend will auch die NPD die Europäische Union als Symbol der politischen Globalisierung aufösen. Sie möchte völlig neue Wege nach Europa beschreiten ${ }^{60}$, ist Anhänger harten Euroskeptizismus. Die EU selbst gilt der NPD als Instrument der Fremdbestimmung. Aus ihrer Sicht haben „die Deutschen“ durch die Europäische Union keine Volkssouveränität mehr. An dieser Stelle wird die NPD widersprüchlich: Sie appelliert an demokratische Prinzipien, die sie sonst gerade ablehnt.

Der Parteivorsitzende Udo Voigt ${ }^{61}$ sieht sich als „Bewegungsunternehmer“62. Er versucht, die NPD in europäische Netzwerke, auch innerhalb der EU, einzugliedern. Seine Partei zückt die internationale Karte, um damit der nationalen Begrenzung zu entgehen. Dieses Unterfangen hat durchaus Erfolg. Am 25. September 2007 konnte die NPD an einem Treffen der rechtsextremistischen Fraktion im Europäischen Parlament in Straßburg teilnehmen. Am Ende stand eine Erklärung für ein „Europa der Vaterländer“63. Inzwischen löste sich die eben erst neu gegründete Fraktion namens ITS ${ }^{64}$ im EP allerdings wieder auf; die ideologischen Divergenzen ließen das Bündnis brüchig werden. Vor allem der Brückenschlag zwischen Rechtsextremisten in West- und Osteuropa missglückte. ${ }^{65}$ Eine Fehde zwischen der italienischen Abgeordneten Alessandra Mussolini und der Groß-Rumänien-Partei führte zum Auszug der rumänischen Abgeordneten. Damit konnte die ITS nicht mehr die für eine Fraktion notwendige Zahl von 20 Abgeordneten erreichen und musste sich auflösen. Die Versuche einer international tragfähigen rechtsextremistischen Kooperation sind daher bislang trotz zunehmender Vernetzung noch nicht gediehen.

Amelie Kutter / Vera Trappmann (Hrsg.), Das Erbe des Beitritts. Europäisierung in Mittel- und Osteuropa, Baden-Baden 2006, S. 171 - 188.

60 Vgl. Selbst Parlamentarier zu „Abnickern“ degradiert. Durch EU-Auflösung zu neuen Wegen nach Europa, in: Deutsche Stimme, 30. Jg. (2006), H. 8, S. 1.

61 Vgl. Eckhard Jesse, Biographisches Porträt: Udo Voigt, in: Uwe Backes / Eckhard Jesse (Hrsg.), Jahrbuch Extremismus \& Demokratie, Bd. 18, Baden-Baden 2006, S. 207 - 219.

62 Vgl. zum Typus des rechtsextremistischen Bewegungsunternehmers: Rainer Erb, Protestorganisation und Eventmanagement: Der Typus des rechtsextremen Bewegungsunternehmers, in: Andreas Klärner / Michael Kohlstruck (Hrsg.), a.a.O. (Fn. 9), S. 142 - 176, S. $142-144$.

63 Vgl. Junge Freiheit vom 5. Oktober 2007, S. 10. Das Organ versteht sich als Brücke zwischen Konservatismus und Rechtsextremismus, lehnt aber die NPD als zu radikal ab.

64 ITS steht für Identität, Tradition und Souveränität.

65 Nach wie vor fehlt es an komparatistischen Studien zwischen dem Rechtsextremismus in Westund Osteuropa. Für Osteuropa gibt es vergleichende Studien, vgl. vor allem die analytisch wegweisende Studie von Tom Thieme, Hammer, Sichel, Hakenkreuz. Parteipolitischer Extremismus in Ostmitteleuropa: Entstehungsbedingungen und Erscheinungsformen, Baden-Baden 2007. Es mangelt aber an detaillierten Einzelfallstudien von Landesexperten. Analytisch ist dieses Unterfangen keineswegs einfach, auch wegen der oftmals fehlenden Abgrenzung zwischen demokratischen und extremistischen Parteien, einschließlich des Wechselspiels zwischen Regierung und Opposition, auch auf der Diskursebene. Ohnehin müsste man dort eine deutliche Trennlinie zwischen Ostmitteleuropa und Südosteuropa ziehen. Für Südosteuropa lässt sich ein sozio-kulturell verankerter Populismus konstatieren. Insgesamt steht die Extremismusforschung hier noch im Anfangsstadium. Vgl. Melani Barlai / Florian Hartleb, Ungarischer Populismus und Rechtsextremismus. Ein Plädoyer für die Einzelfallforschung, in: Südosteuropa Mitteilungen, 49. Jg. (2008), H. 4, S. $34-51$. 


\subsection{Kulturelle Dimension}

Rechtsextremisten lehnen die kulturelle Globalisierung ab. Nach der von ihnen aufgegriffenen Homogenisierungsthese ${ }^{66}$ ist die Globalisierung ein eindimensionaler Prozess, Ausdruck von Massenkultur und Massengeschmack, Degeneration und Verflachung. Durch die (sub-)kulturelle Dominanz der USA kommt ein vielfältig aufgeladener Antiamerikanismus als Gegenpol ins Spiel. Amerikaressentiments können dabei verschiedene Facetten haben, antikapitalistisch, antichristlich, menschenrechtsorthodox und eben auch kulturell bedingt sein. Ein gängiges Stichwort lautet McDonaldisierung als Symbol für kulturelle Verflachung und den American way of life. ${ }^{67}$

Ein fundamentaler Antiamerikanismus nimmt auch bei der NPD breiten Raum ein. Sie warnt vor Amerikanisierung, kultureller und ethnischer „Überfremdung“, dem „melting pot USA“. Fremde Kulturen dürfen ihr zufolge nicht auf die Nationalkultur wirken. Die „Globalisten“ und „Multikulturalisten“ firmieren als Feindbilder, die sich für Verschwörungstheorien aller Couleurs eignen. Die NPD vertritt die rassistisch-ethnische Homogenitätsposition, mit den USA als Negativfolie. Sie suggeriert, für etwas kulturell „Höherwertiges“ zu stehen, propagiert aber undifferenziert eine Blut- und Boden-Kultur und negiert jegliche Transzendenz. Diese Spannung zwischen Immanenz und Überweltlichkeit ermöglichte aber letztlich die „abendländische Kultur“. Damit argumentiert die NPD doppelzüngig.

Insgesamt setzt sich die NPD im Antiglobalisierungsdiskurs scharf von den etablierten Parteien ab. Dabei differenziert sie zwischen dem Prozess der Globalisierung und dem Globalismus. In „12 Thesen zum Globalismus“ heißt es auf ihrer Homepage: „Globalisierung ist der Prozess, dessen sich die Globalisten zur Durchsetzung ihrer Ziele bedienen. Die von den Globalisten hervorgerufenen Migrationsströme führen [...] zur Zerstörung gewachsener Sprachen und Kulturen. "68 Damit wird das von der NPD gezeichnete Bild klar: Der Prozess der Globalisierung vernichtet Kulturen, Traditionen und Werte. Am Ende gehen ihr zufolge ganze Nationen und Völker unter: „Die etablierten Politiker beschwören durch ihre Zuwanderungspolitik den Völkerselbstmord herbei. Der kapitalistisch diktierte Bevölkerungsaustausch hat in letzter Konsequenz Völkermordcharakter. “69

Die „neue“ NPD trägt Züge einer neuen sozialen Bewegung, allerdings mit begrenzter Reichweite und Resonanz.

66 Neben dem Homogenisierungsvorwurf existieren aus wissenschaftlicher Perspektive andere kulturalistische Globalisierungstheorien. So ist häufig auch von einer Kreolisierung oder Hybridisierung von Kulturen die Rede. Bedeutung hat auch der Begriff der Glokalisierung erlangt. Vgl. Bernd Wagner, Kulturelle Globalisierung: Weltkultur, Glokalität und Hybridierung, in: ders. (Hrsg.), Kulturelle Globalisierung. Zwischen Weltkultur und kultureller Fragmentierung, Frankfurt am Main / Essen 2001, S. 9 - 38.

67 Vgl. zur systematischen Bestimmung von Amerikaressentiments Lazaros Miliopoulos, Die Verneinung des atlantischen Zivilisationszusammenhangs und der Extremismus. Ideengeschichtliche Topoi einer Wirklichkeitsverkennung, in: Uwe Backes / Eckhard Jesse (Hrsg.), Jahrbuch Extremismus \& Demokratie, Bd. 19, Baden-Baden 2007, S. 35 - 65.

68 Vgl. www.npd.de/npd_programme/12_thesen_zum_globalismus.html (Abruf am 10. September 2008).

69 Vgl. Jürgen W. Gansel, Mitteldeutschland als Testfall der Globalisierer. Der Teufelskreislauf aus Arbeitslosigkeit, Abwanderung und Hoffnungslosigkeit, in: Deutsche Stimme, 30. Jg. (2006), H. 8, S. 9. 


\section{NPD: keine (Anti-)Globalisierungsbewegung}

Die NPD, jahrzehntelang eine altrechte, selbst innerhalb des rechtsextremen Spektrums bedeutungslose Partei, ist modernisiert und in programmatischer Hinsicht internationalisiert. Sie greift seit geraumer Zeit zu einem Anti-Globalisierungsdiskurs, um sich als eine „neue soziale Bewegung“ stilisieren zu können. Gleichwohl sind der Partei Grenzen gesetzt: So ist im bundesdeutschen Parteienwettbewerb eine Nationalismus-Globalismus-Konflikt$\operatorname{linie}^{70}$ kaum ausgeprägt und wegen zahlreicher aktueller hausgemachter Probleme der NPD auch wenig wahrscheinlich. ${ }^{71}$ Für Mecklenburg-Vorpommern und Sachsen fällt das Urteil durch die parlamentarische Präsenz der NPD und ihrer Verbindung zu den „freien Kräften“ anders aus. Auch die Szenarien der NPD von einer lagerübergreifenden Globalisierungskritik finden keine reale Grundlage - vor allem, weil die linke wie auch die linksextremistische $^{72}$ Globalisierungskritik sich vom rechtsextremistischen Antiglobalisierungsgestus scharf distanziert. Die praktische Umsetzung der Globalisierungskritik wie beim G8-Gipfel in Heiligendamm ist nicht in Sicht. Parlamentarische Initiativen wie die Forderung nach Einführung eines Regionalgeldes in Mecklenburg-Vorpommern entfalten wenig Attraktivität - sogar im eigenen Lager. ${ }^{73}$

Die NPD kann deshalb ihr Ziel einer tragfähigen (Anti-)Globalisierungsbewegung nicht erreichen. Allerdings spielt sie auf der Klaviatur des Globalisierungsdiskurses. Dieser neuen Argumentation „jenseits des Nationalstaats“ sollte daher in Öffentlichkeit wie Forschung genauer Rechnung getragen werden. Internationale Entwicklungen im Rechtsextremismus ${ }^{74}$ lassen auf einen neuen, bewegungsförmigen Faschismus im Verbund schließen. Weniger bloße Wahlergebnisse, vielmehr die Beobachtung der rechtsextremen „Demonstrationspolitik“ und die Bewertung des programmatischen Diskurses ermöglichen eine Bilanz des Rechtsextremismus in Europa.

70 Das sehen für Deutschland folgende Autoren: Susanne Frölich-Steffen / Lars Rensmann, Conditions for failure and success of right-wing populist parties in public office in the New European Union, in: Pascal Delwit / Philippe Poirier (Hrsg.): Extrême droite et pouvoir en Europe. The extreme right parties and power in Europe, Brüssel 2007, S. 117 - 139, S.124.

71 Dazu zählen aktuelle finanzielle Probleme, die aus Rückzahlungsansprüchen nach fingierten Abrechnungen resultieren. Eine neue Welle von schlechten Wahlergebnissen sorgt auch für Kritik an der Parteiführung und Udo Voigt und Unruhe im Verhältnis zur DVU und zu den „freien Kräften".

72 Hier sind besonders autonome Gruppierungen angesprochen, wie der gewalttätige „Schwarze Block“.

73 Vgl. Kevin Stützel, Antikapitalismus von rechts? Globalisierungskritik, die extreme Rechte und der G-8-Gipfel in Heiligendamm, in: rls Standpunkte 2007, H. 13, S. 1 - 9, S. 8.

74 Vgl. exemplarisch zu den Entwicklungen in Ungarn und der Entstehung einer „paramilitärischen Garde“ als „neue soziale Bewegung“ Melani Barlai / Florian Hartleb, a.a.O (Fn. 65). 